\title{
Diseño y validación de contenido de un instrumento para medir la gestión de la seguridad y salud en el trabajo para organizaciones colombianas $^{*}$
}

\section{Content design and validation of an instrument to measure safety and health management at work for Colombian organizations}

Recibido: 20 de diciembre de 2015 Revisado: 30 de junio de 2016 Aceptado: 1 de agosto de 2016

Gloria María Erazo Imbacuán***

Colmena

Yuber Liliana Rodrígue*-Rojas****

Convenio Universidad Santo Tomás - ICONTEC

\section{RESUMEN}

En Colombia, ante la gran expectativa y evolución de las normativas emanadas en la Ley 1562 de 2012 y en el Decreto 1072 de 2015, es preciso construir y aplicar indicadores válidos y legitimados, pues los indicadores son la visión compartida de la forma como se logran los fines. Por lo anterior, en esta investigación se diseñó un instrumento para medir la gestión de la seguridad y salud en el trabajo con validez de

* Artículo de resultado de investigación.

* Ingeniera Biomédica. Magister en Calidad y Gestión Integral. Coordinadora técnica nacional, Colmena. Correo electrónico: ing.gloria.maria. erazo@gmail.com

*** Fisioterapeuta. Magister en Salud y Seguridad en el Trabajo. Doctorado en curso en Administración. Docente Maestría en Calidad y Gestión Integral Convenio Universidad Santo Tomás - ICONTEC. Correo e: yuberliliana@gmail.com. 
contenido para organizaciones colombianas, bajo la estructura de alto nivel planteada por la ISO, la cual será la base de la futura norma técnica ISO 45001. A partir de la revisión de la literatura y la consulta a expertos (14 expertos de 6 administradoras de riesgos laborales) se construyó el instrumento, el cual integró y alineó los requerimientos de cumplimiento del Decreto 1072 de 2015 y del draft de la norma técnica ISO 45001, desde el principio de que el instrumento es una herramienta que provee información para la toma de decisiones en materia de prevención y control de riesgos.

Palabras clave: gestión, instrumentos de medición, seguridad y salud en el trabajo, validación de contenido.

\section{ABSTRACT}

In Colombia, given the great expectation and evolution of the regulations issued in Law 1562 of 2012 and in Decree 1072 of 2015. For this reason, it is necessary to construct and apply valid and legitimized indicators, since the indicators are the shared vision of the form in That the ends are achieved. This research was designed as an instrument to measure the Safety and Health Management at Work with content validity for Colombian organizations under the high level structure proposed by ISO, which will be the basis of the future ISO 45001. From the review of the literature and the consultation of experts (14 experts from six Occupational Risk Managers) the instrument was built, which integrated and aligned the compliance requirements of Decree 1072 of 2015 and Draft of ISO 45001, Under the principle that the instrument is a tool that provides information for decision making in the prevention and control of risks.
Keywords: Management, Measuring Instruments, Safety and Health at Work, content validation.

\section{INTRODUCCIÓN}

El mejoramiento de la gestión de la seguridad y salud en el trabajo (GSST) en las organizaciones es un elemento de gran importancia para lograr los niveles de calidad y productividad requeridos en los momentos actuales de un mundo globalizado. Este proceso necesita diagnóstico sistemático para la elaboración de planes de acción que permitan la eliminación de los problemas existentes en este campo en las organizaciones.

Desde esta perspectiva, los dos factores claves del éxito para el verdadero desarrollo de la prevención en las organizaciones están dados por una fuerte integración de la salud y seguridad en el funcionamiento de la empresa y la gestión de los riesgos centrada en las situaciones de trabajo (Favaro y Drais, 2007). Desde esta dimensión, la GSST refleja un estado de madurez de las organizaciones frente al tema de la prevención, la cual se evidencia en el nivel de posicionamiento que tiene dentro de su estructura. Aunado a ello, estos sistemas de gestión están influenciados por aspectos normativos y por la concepción de la prevención (Favaro, 2006).

En relación con la seguridad y salud en el trabajo (SST), también se evidencia un desarrollo en la conceptualización de salud-enfermedad en el marco de la trasformación de la salud ocupacional hacia modelos de planeación y seguimiento en torno a la calidad y a la gestión de la seguridad y la salud en el trabajo, que permitan realizar acciones de mejora y diseño de nuevas estrategias en materia de promoción y prevención, con el objeto de mejorar la calidad de vida de los trabajadores (Rodríguez, 2012). 
Así es como uno de los aspectos de importancia en relación con la GSST es la medición, para lo cual se evidencia un incipiente avance en el diseño de herramientas que abarquen variables jerárquicas organizacionales; sin embargo, existen intervalos vacíos a nivel teórico y conceptual, en cuanto a los procesos que no solo impactan el hacer, sino también la evaluación de indicadores.

En este sentido, la aplicación de herramientas de medición en el marco de un sistema dinámico organizacional de GSST integra la necesidad de las organizaciones de alcanzar sus objetivos de sostenibilidad, crecimiento y rentabilidad laboral y de sus trabajadores, pues la evaluación, el seguimiento y control llevan a una toma de decisiones oportuna y adecuada referente a procesos, procedimientos y recursos que puedan ser intervenidos para mejorar la calidad de vida de los trabajadores. Sin embargo, cabe resaltar que aunque se han logrado avances en materia de evaluación, son evidentes los vacíos teóricos y conceptuales en relación con la gestión y la seguridad en el trabajo.

El progreso histórico del cual datan los registros corresponden a los últimos veinte años, en los cuales se evidencia un escaso avance sobre investigaciones consolidadas en torno al desarrollo de herramientas y estrategias que den respuesta a conceptos evaluativos más cercanos a los requerimientos que el mundo en la actualidad exige frente a las relaciones laborales y al equilibrio entre la rentabilidad y sostenibilidad organizacional, la protección de los trabajadores y la seguridad y la salud en el trabajo.

Por otra parte, al revisar la literatura previa a la los años noventa se encuentra que no existe un sistema de GSST normalizado y reconocido en el mundo. En 1999 se publicó la OHSAS 18001:1999 sobre sistemas de gestión en seguridad y salud ocupacional; por esta razón, cada organización, antes de esta fecha, generaba sus propios modelos o sistemas de gestión, dada la complejidad de sus procesos de trabajo y sus necesidades, y atendiendo a los requirimiento legales de cada país (Fernández, 2007; Fernández, Montes y Vázquez, 2006). Este fenómeno se debe a que antes los esfuerzos de seguridad se consideraban acciones aisladas y se centraban es aspectos técnicos, y se dejaban de lado los organizativos y humanos; sin embargo, en los últimos años se han considerado los factores organizacionales, culturales y de gestión que desempeñan un papel primordial en la generación de accidentes e incidentes.

Por otro lado, se puede afirmar que lo que ha dificultado la adopción de estándares internacionales es la falta de ellos en algunos países industrializados y la carencia de investigaciones en materia de la GSST. Por consiguiente, si bien existen diferentes modelos, sus diferencias sustanciales radican en la posibilidad de certificación y también en su influencia en la voluntariedad, así como en el nivel de desarrollo o grado de especificación de este, lo que subyace en los intereses políticos y económicos de la organización (Rubio, 2001).

En el caso particular de Colombia, es necesario el desarrollo de instrumentos de medición de GSST que sean flexibles para la gran, mediana y pequeña empresa, incluidos trabajadores independientes, que deben ser aplicadas a la luz de la Ley 1562 de 2012 y del Decreto 1072 de 2015 por todos los empleadores públicos y privados, los contratantes de personal con modalidad de contrato civil, comercial o administrativo, las organizaciones de economía solidaria y del sector cooperativo, las organizaciones de servicios temporales y tener cobertura sobre los trabajadores dependientes, contratistas, trabajadores cooperados y los trabajadores en misión. 
Figura 1. Perspectiva histórica de los sistemas de gestión de la salud y seguridad

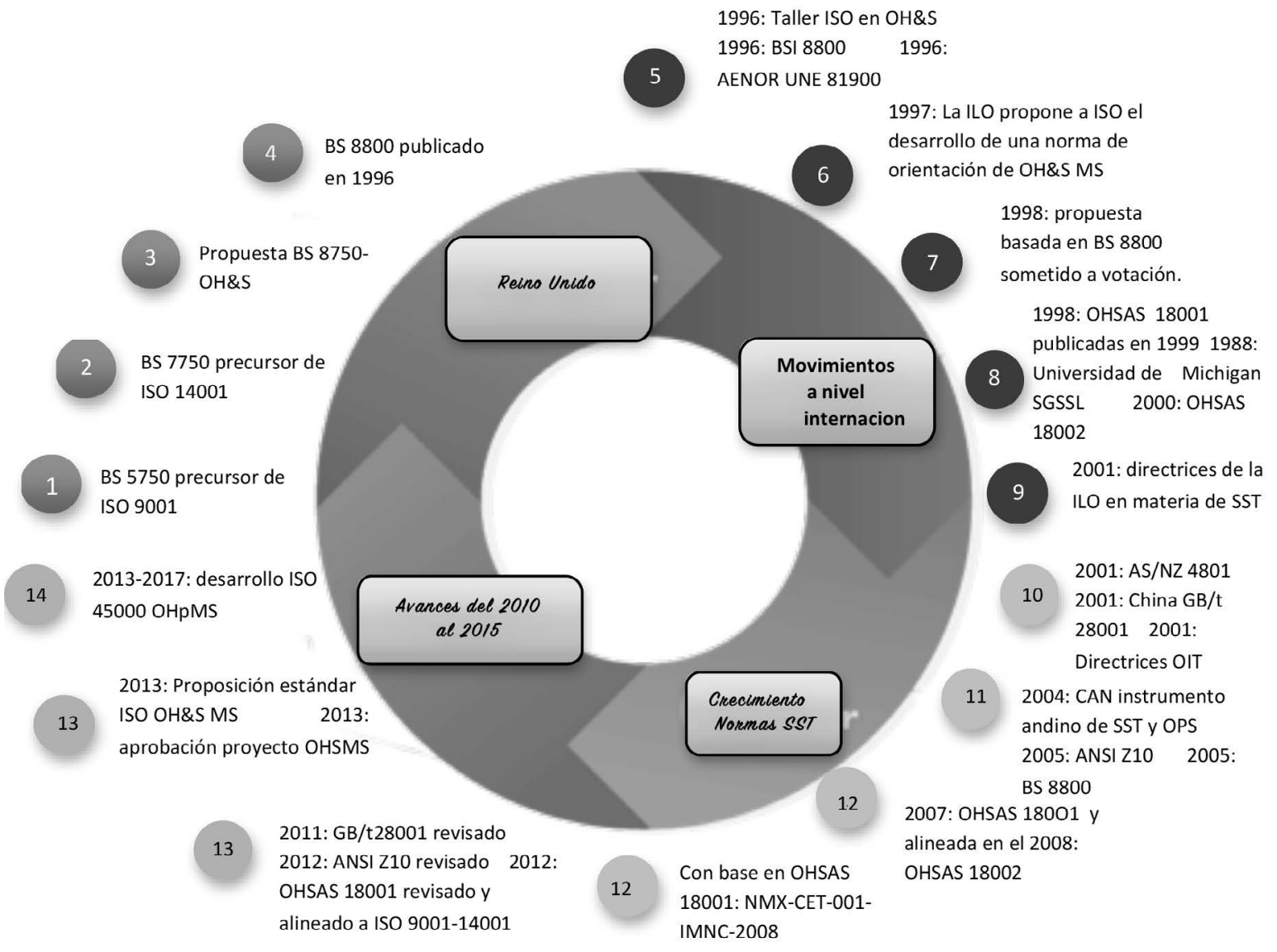

Fuente: elaboración propia, a partir del DRAFT ISO 45001 y la interpretación de Glaesel, K.

\section{Antecedentes del contexto en Colombia}

La GSST en Colombia es un campo de exploración naciente, en cuanto a nivel gubernamental. Solo hasta cuando se emanó la Ley 1562 de 2012, por la cual se modificó el sistema de riesgos laborales y se dictaron otras disposiciones en materia de salud ocupacional, se evidenció la evolución en el tema, pues se concibió con el objetivo principal de lograr una mayor cobertura respecto a la protección de los trabajadores formales e informales y se amplió así el espectro empresarial.

El Decreto 1443 de 2012, derogado por el Decreto 1072 de 2015, se convirtió en la primera orientación estándar que data la intención de dar inicio al proceso de medición de GSST en Colombia, y de acuerdo con el artículo 37 "Transición",

Todos los empleadores deberán sustituir el Programa de Salud Ocupacional por el Sistema de Gestión de la Seguridad y Salud en el Trabajo (SGSST) para lo cual, a partir de la publicación del presente Decreto deberán dar inicio a las acciones necesarias para ajustarse a lo establecido en esta disposición y tendrán unos plazos para culminar la totalidad del proceso, contados a partir de la entrada en vigencia del presente Decreto, de la siguiente manera: Dieciocho (18) meses para las organizaciones con menos de diez (10) trabajadores, Veinticuatro (24) meses 
para las organizaciones con diez (10) a doscientos (200) trabajadores y Treinta (30) meses para las organizaciones de doscientos uno (201) o más trabajadores. (Decreto 1443, 2014)

La norma, al ser tan reciente, aún está en proceso de cumplimiento, razón por la cual todavía no existen registros documentados o resultados en la aplicación del sistema.

Ante esta realidad, las Administradores de Riesgos Laborales (ARL) en Colombia inician los procesos de asesoría técnica en el tema diseñando, a la luz del Decreto 1443 y posteriormente del Decreto 1072 de 2015, herramientas de medición internas para sus clientes, todas ellas soportadas en las directrices de la Organización Internacional del Trabajo (OIT, 2001). Estas asesorías se realizan con una labor conjunta en la cual participa la alta gerencia, los equipos de SST, Comités Paritarios de Seguridad y Salud en el Trabajo (COPASST) y asesores de las ARL, que capacitan, entrenan y guían los ejercicios de evaluación de las condiciones existentes de las organizaciones en la materia, para que los empleadores tengan la información para implementar el sistema a través de la aplicación de un instrumento que cuenta con 19 criterios estándar y 152 ítems de validación.

Con la inminente evolución en materia legal en Colombia sobre la medición en GSST, los desarrollos investigativos frente a la validación de instrumentos que permitan realizar esta acción inician su proceso, del cual el único referente en su contexto teórico y de resultados es la adaptación de una herramienta para la evaluación de la GSST, que buscó identificar y adaptar un instrumento francés al contexto colombiano, con el fin de garantizar el rigor científico en su creación y aplicación en la evaluación de la GSST. Esto, atendiendo a la metodología propuesta por Beaton, en la cual se alcanzaron dos resultados de interés: en primer lugar, comprobar que la GSST ha sido poco estudiada en el país; así, esta se reconoce como un factor orientador e integrador de la protección de la salud y el fomento de la seguridad en las actividades cotidianas de una organización, que contribuye, además, al éxito y la sostenibilidad del negocio; en segundo lugar, generar una versión del instrumento semánticamente similar al de origen francés. Para esto se efectuó la traducción respectiva al español, la revisión técnica por parte de un experto, tanto en el contexto francés como en el colombiano y de verificación de expresiones técnicas (Rodríguez y Molano, 2012).

Después de haber realizado la adaptación, se detectaron para el contexto colombiano algunas brechas; por ejemplo, al realizar la aplicación cobra un fuerte requerimiento la experticia, experiencia y dominio técnico de quien aplique el instrumento; sin embargo, en Colombia la segmentación empresarial para micro, pequeñas y medianas organizaciones no cuenta en su totalidad con personal especializado en materia de SST y GSST. De hecho, el manejo se ve compartido con las áreas de talento humano, donde el personal solo recibe apoyo técnico de la ARL. De igual modo, este instrumento es aplicado en los niveles estratégico y táctico, y no involucra dentro de la evaluación de la GSST la participación directa de los trabajadores, lo que puede generar sesgos en la información recopilada (Rodríguez y Molano, 2002).

En Colombia, al igual que en Latinoamérica, el estudio del riesgo psicosocial tiene gran importancia y relevancia, y por ello los antecedentes y referentes a desarrollos investigativos en este campo son más predominantes en comparación con la investigación sobre gestión en seguridad y salud en el trabajo.

Por los anteriores supuestos, se requiere entonces pensar en un modelo de GSST que vaya más allá del cumplimiento legal, que permita contribuir a la mejora de la gestión de las organizaciones y que incluya la 
responsabilidad social; además, debe basarse en el sistema legal de cada país, la propuesta de las directrices de la OIT, la norma OHSAS:2007 y la guía para la elaboración de memorias de sostenibilidad (corresponde a descripción de los impactos de las actividades de una organización en los sistemas sociales donde opera. Esta guía sirve para describir por parte de la organización los resultados de la adopción y aplicación de códigos, políticas y sistemas de gestión [Salinas, 2008]).

\section{METODOLOGÍA}

Esta investigación es de tipo cuantitativo, puesto que diseñó un instrumento basado en el draft de la norma técnica ISO 45001. Para esto se definieron los parámetros de evaluación de la GSST a través de la revisión bibliográfica de instrumentos existentes en las bases de datos especializadas.

El proyecto en su etapa de diseño contó con una propuesta de instrumento basado en el Draft de la ISO 45001 y se definieron los parámetros de evaluación de la GSST, a través de la revisión bibliográfica de instrumentos existentes en las bases de datos MedlinePubmed, Cochrane, Science Direct y BVS, teniendo en cuenta los siguientes parámetros de búsqueda MESH: occupational health (salud ocupacional), management (gestión), organiæation (organización), administration (administración), safety (seguridad) y manpower (recursos humanos) y las ecuaciones de búsqueda Occupational health and management, Organisation and administration, Occupational health and safety and management, Occupational and health and manpower.

Como resultado de esta revisión bibliográfica y dadas las condiciones técnicas, se establecieron los parámetros de análisis y se desarrollaron los comparativos estructurales de los instrumentos existentes. Por ello, se determinó que el instrumento que contaba con las características de validación de constructo y contenido era el francés Gestión de la Santé el de la Ségurité Au Travial Dans L'Entreprise, que fue usado como base documental para el diseño y se alineó con los parámetros de obligatorio cumplimiento del Decreto 1072 de 2015, en lo referente a la SST; adicionalmente, se realizó el comparativo con la estructura del Draft de la ISO 45001.

Al hacer el cruce de variables de los instrumentos se evidencia la tendencia transversal por la búsqueda de la mejora continua; se aprecia como elemento común el PHVA, por lo tanto se tomó este modelo como base para el análisis.

Otro aspecto relevante en estas estructuras es el énfasis en el seguimiento y el control, pues todas cumplen con auditorías internas y externas como mecanismo para realizar evaluaciones permanentes que permitan, en el proceso de implementación del sistema de gestión y de los planes de acción prioritaria, el ajuste oportuno de indicadores para el cumplimiento de metas.

A pesar de que las estructuras son homólogas en su fundamentación, también existen diferencias marcadas, como la obligatoriedad para la implementación del Decreto 1072 de 2015 en comparación con la voluntariedad para la ISO 45001 y el instrumento de la Gestion de la Santé de la Ségurité Au travail Dans L"Entreprise.

Un aspecto diferencial del comparativo está dado en dos variables, que el draft de la norma técnica ISO 45001 especifica referente hacia la medición de variables sobre cultura organizacional y participación de los trabajadores, y el concepto general acerca de que la organización debe examinar sus riesgos de negocio en el desarrollo de su sistema de gestión y utilizarlo como una herramienta para evitar que se produzca el riesgo. 
En la etapa de validación de contenido el instrumento de medición fue sometido a un proceso de revisión, el cual generó aportes técnicos sobre la eliminación de ítems que por su concepto no eran aplicables a la realidad colombiana, de acuerdo con el Decreto 1072 de
2015. Ítems que por su redacción eran repetitivos en su estructura y significado, en un pilotaje por lo cual se tuvo en cuenta el juicio experto de catorce profesionales técnicos de seis ARL del país (su perfil se describe en la tabla 1).

Tabla 1. Perfiles profesionales y laborales de los expertos

\begin{tabular}{|c|c|c|}
\hline CARGO & PERFIL PROFESIONAL & EXPERIENCIA \\
\hline \multirow{4}{*}{$\begin{array}{l}\text { Asesor externo Profesional en Riesgos } \\
\text { Laborales }\end{array}$} & Psicólogo & \multirow{4}{*}{15 años } \\
\hline & Especialista de Salud Ocupacional & \\
\hline & Maestrante de Planeación Estratégica y Gestión del Conocimiento & \\
\hline & Docente Universidad del Tolima & \\
\hline \multirow{4}{*}{ Asesora externa } & Fonoaudióloga & \multirow{4}{*}{10 años } \\
\hline & Especialista en Salud Ocupacional & \\
\hline & Maestrante de Planeación Estratégica y Gestión del conocimiento & \\
\hline & Docente Universidad del Tolima & \\
\hline \multirow[t]{2}{*}{ Asesor empresa } & Psicóloga & \multirow{2}{*}{4 años } \\
\hline & Especialista en Seguridad y Salud en el Trabajo & \\
\hline \multirow{2}{*}{ Director integral de servicios I } & Administradora de Empresas & \multirow{2}{*}{8 años } \\
\hline & Especialista en Higiene y Salud Ocupacional & \\
\hline \multirow{3}{*}{ Asesor en prevención $\mathrm{V}$} & Psicólogo & \multirow{3}{*}{10 años } \\
\hline & Especialista en Salud Ocupacional & \\
\hline & Especialista en Higiene Industrial & \\
\hline \multirow{6}{*}{ Asesor en prevención VI } & Psicóloga & \multirow{6}{*}{10 años } \\
\hline & Especialista en Gerencia y Salud Ocupacional. & \\
\hline & Especialista en Pedagogía y Docencia Universitaria & \\
\hline & Especialista en Educación y Orientación Familiar & \\
\hline & Docente de la Fundación Universitaria Monserrate & \\
\hline & Docente Universidad Externado de Colombia & \\
\hline
\end{tabular}




\begin{tabular}{|c|c|c|}
\hline CARGO & PERFIL PROFESIONAL & EXPERIENCIA \\
\hline \multirow{3}{*}{ Asesor en prevención por proyecto VI } & Fisioterapeuta & \multirow{3}{*}{10 años } \\
\hline & Especialista en Gerencia de Salud Ocupacional & \\
\hline & Estudiantes Derecho VI semestre & \\
\hline \multirow{2}{*}{ Líder de productos y servicios } & Ingeniero Ambiental & \multirow{2}{*}{7 años } \\
\hline & Especialista en Seguridad Industrial, Higiene y Gestión Ambiental & \\
\hline \multirow{2}{*}{ Director integral de servicios Master } & Ingeniero Industrial, Especialista en Salud Ocupacional & \multirow{2}{*}{12 años } \\
\hline & Docente universitario & \\
\hline \multirow{2}{*}{ Asesor integral de servicios VIII } & Psicóloga & \multirow{2}{*}{12 años } \\
\hline & Especialista en Salud Ocupacional & \\
\hline \multirow{3}{*}{ Gestor gestión } & Ingeniero Industrial & \multirow{3}{*}{4 años } \\
\hline & Especialista en la Gerencia de la Seguridad y Salud en el Trabajo & \\
\hline & Auditor Interno HSEQ & \\
\hline \multirow{3}{*}{ Director integral de servicios I } & Psicólogo & \multirow{3}{*}{7 años } \\
\hline & Especialista en Psicología de la Salud Ocupacional & \\
\hline & Auditor Interno en Sistemas Integrados de Gestión & \\
\hline \multirow{3}{*}{$\begin{array}{l}\text { Profesional especializado para riesgo } \\
\text { biomecánico }\end{array}$} & Fisioterapeuta & \multirow{3}{*}{15 años } \\
\hline & Especialista en Gerencia de Salud Ocupacional & \\
\hline & Master en Sistemas Integrados de Gestión de Calidad & \\
\hline Asesora en prevención & Profesional en Seguridad Industrial y Salud Ocupacional & 16 años \\
\hline
\end{tabular}

Fuente: elaboración propia.

\section{RESULTADOS Y DISCUSIÓN}

En esta sección se presenta, en la primera parte, la estructura del instrumento para la medición de la GSST propuesto para organizaciones colombianas y su escala de califación. En la segunda parte, se describen los resultados de la validación del contenido del instrumento realizada con 14 expertos vinculados a seis ARL de Colombia.

\section{Estructura del intrumento y escala de medición}

Para el desarrollo del instrumento se tomaron como estructura organizadora las dimensiones de la estructura de alto nivel planteada por la ISO, la cual será la base de la futura ISO 45001. Cabe precisar que a partir del análisis de los requisitos establecidos en el Decreto 1072 de 2015 y los propuestos en el Draf de la ISO 
45001 se identificó que son compatibles si se analizan desde la estructura de alto nivel mencionada. En la tabla 2 se presentan las dimensiones de la estructura de alto nivel y los criterios específicos asociados a cada dimensión para el caso de la GSST, aún denominada en el Draft como gestión de la segruidad y salud ocupacional. Por consiguiente, el instrumento diseñado está compuesto por las 6 dimensiones y 30 criterios descritos en esta tabla 2.

Tabla 2. Dimensiones Draft ISO 45001

\begin{tabular}{|c|c|}
\hline DIMENSIONES & CRITERIO \\
\hline \multirow{8}{*}{ Contexto de la organización } & Entendimiento del contexto de la organización \\
\hline & Comprender las necesidades y expectativas de los trabajadores y otras partes interesadas \\
\hline & Alcance del sistema de gestión de S \& SO \\
\hline & $\mathrm{OH} \& \mathrm{~S}$ del sistema de gestión \\
\hline & Liderazgo, participación de los trabajadores y la consulta \\
\hline & Política \\
\hline & Organización de roles, responsabilidades y autoridades \\
\hline & La participación, la consulta y la representación \\
\hline \multirow{3}{*}{ Planeación } & Acciones para hacer frente a los riesgos y oportunidades \\
\hline & Identificación y evaluación de riesgos de S \& SO de Peligros \\
\hline & Identificación de peligros \\
\hline \multirow{5}{*}{ Apoyo } & Recursos \\
\hline & Competencia \\
\hline & Conciencia \\
\hline & Información y comunicación \\
\hline & Documentación de información \\
\hline \multirow{6}{*}{ Operación } & Planeación y controles operacionales \\
\hline & Jerarquía de controles \\
\hline & Gestión del cambio \\
\hline & Subcontratación \\
\hline & Contratación \\
\hline & Preparación ante emergencias \\
\hline \multirow{3}{*}{ Evaluación de desempeño } & Monitoreo, medición, análisis y evaluación \\
\hline & Método \\
\hline & Evaluación de rendimiento \\
\hline
\end{tabular}




\begin{tabular}{|l|l|}
\hline \multicolumn{1}{|c|}{ DIMENSIONES } & \multicolumn{1}{c|}{ CRITERIO } \\
\hline \multirow{4}{*}{ Evaluación de desempeño } & Evaluación de cumplimiento \\
\cline { 2 - 2 } & Auditoria externa \\
\cline { 2 - 2 } & Auditoria interna \\
\cline { 2 - 2 } & Revisión por la dirección \\
\hline \multirow{2}{*}{ Mejora } & Incidentes, inconformidad y correctivo de acción \\
\cline { 2 - 2 } & Mejora continua \\
\hline
\end{tabular}

Fuente: elaboración propia a partir de la ISO/CD 45001.2 Occupational health and safety management systems - Requirements with guidance for use (Systèmes de management de la santé et de la sécurité au travail — Exigences et lignes directrices pour son utilisation).

Las seis dimensiones referidas cuentan con su despliegue funcional referenciado en sus ítems de evaluación, variables, estándares, numerales de identificación, criterios e items de verificación, como se expone en la tabla 3.

Tabla 3. Descripción cuantitativa de despliegue funcional

\begin{tabular}{|l|c|c|c|c|c|c|}
\hline \multirow{2}{*}{\multicolumn{1}{|c|}{ DESCRIPCIÓN }} & \multicolumn{6}{|c|}{ ETAPAS } \\
\cline { 2 - 8 } & 1 & 2 & 3 & 4 & 5 & 6 \\
\hline Dimensión & 1 & 1 & 1 & 1 & 1 & 1 \\
\hline Ítem de evaluación & 5 & 2 & 4 & 4 & 4 & 2 \\
\hline Variables & 3 & 2 & 7 & 4 & 7 & 2 \\
\hline Estándares & 12 & 3 & 7 & 4 & 7 & 2 \\
\hline $\begin{array}{l}\text { Numerales de } \\
\text { identificación }\end{array}$ & 44 & 24 & 20 & 20 & 25 & 7 \\
\hline Criterios & 44 & 24 & 20 & 20 & 25 & 7 \\
\hline Ítems de verificación & 44 & 24 & 20 & 20 & 25 & 7 \\
\hline
\end{tabular}

Fuente: elaboración propia.

El procedimiento de escalamiento de los cuantificadores lingüísticos del instrumento estará dado por la escala de Likert 1-4 en función de las siguientes ventajas: "Menos ambigüedad de respuestas al objetivo de investigación, permiten recabar más información en menor tiempo, sencilla de responder y rápida de contestar" (Cañadas y Sánchez, 1998, p. 623).
Para este caso, cada ítem se evaluará de acuerdo con la escala descrita en la tabla 4.

Tabla 4. Escala de evaluación del instrumento

\begin{tabular}{|c|l|}
\hline ABREVIATURA & \multicolumn{1}{|c|}{ SIGNIFICADO } \\
\hline CT & Cumple totalmente \\
\hline CP & Cumple parcialmente \\
\hline NC & No cumple \\
\hline NA & No aplica \\
\hline
\end{tabular}

Fuente: elaboración propia.

Con el fin de ponderar el peso de cada dimensión, se tendrá en cuenta el porcentaje de cumplimiento presentado en la tabla 5 con un 0,4 margen de error.

Tabla 5. Escala porcentual de cumplimiento

\begin{tabular}{|l|c|}
\hline \multicolumn{1}{|c|}{ DIMENSIÓN } & \% DE CUMPLIMIENTO \\
\hline Contexto de la Organización & 5 \\
\hline Planeación & 18,92 \\
\hline Apoyo & 18,92 \\
\hline Operación & 18,92 \\
\hline Evaluación de desempeño & 18,92 \\
\hline Mejoramiento & 18,92 \\
\hline
\end{tabular}

Fuente: elaboración propia. 
Los resultados de la aplicación del instrumento se realizarán a través de histogramas tipo radar, de acuerdo con los estudios realizados por Drais, Favaro y Aubertain (2008) y Rodríguez y Molano (2012)

[...] para representar los resultados, se recomienda usar gráficos tipo histograma 0 radar con escala de 0 a 10 . Este tipo de representación gráfica permite identificar fácilmente las vías de progreso y de igual modo comparar las empresas que conforman un grupo empresarial; a su vez, el promedio de estos datos da cuenta de la situación global del grupo de empresas. (p. 14)

Ahora bien, adaptando este concepto al instrumento diseñado, se tendrán las siguientes puntuaciones para cada una de las dimensiones, mencionadas en la tabla 6.

Tabla 6. Puntuación escala de evaluación

\begin{tabular}{|l|c|c|c|}
\hline \multicolumn{1}{|c|}{ DIMENSIÓN } & CRITERIOS & $\begin{array}{c}\text { UNIDAD } \\
\text { PUNTUACIÓN }\end{array}$ & $\begin{array}{c}\text { PUNTUACIÓN } \\
\text { TOTAL }\end{array}$ \\
\hline Contexto de la organización & 44 & 0,22 & 10 \\
\hline Planeación & 24 & 1,57 & 37,84 \\
\hline Apoyo & 20 & 1,89 & 37,84 \\
\hline Operación & 20 & 1,89 & 37,84 \\
\hline Evaluación de desempeño & 25 & 1,51 & 37,84 \\
\hline Mejoramiento & 7 & 5,40 & 37,84 \\
\hline Total & 140 & 140 & 199,2 \\
\hline
\end{tabular}

Fuente: elaboración propia. 


\section{Resultados de la validación de contenido}

A partir de la evauación por los 14 expertos, el instrumento se ajustó realizando modificaciones en su redacción y estructura, de acuerdo con el resultado de la aplicación de las encuestas que se presenta en las tablas 4 y 5.

Tabla 4. Resultados de la evaluación efectuada por parte de los expertos al instrumento

\begin{tabular}{|c|c|c|}
\hline No. & PREGUNTA & RESULTADOS CONSOLIDADOS \\
\hline 1 & $\begin{array}{l}\text { ¿El instrumento da respuesta a la evaluación de todos } \\
\text { los parámetros que el Decreto } 1072 \text { de } 2015 \text { indica } \\
\text { que son de obligatorio cumplimiento en Colombia } \\
\text { para la SST? }\end{array}$ & $\begin{array}{l}\text { Para este ítem se obtuvo un } 64,28 \% \text { de cumplimiento. En el instrumento fue } \\
\text { necesario hacer ajustes sobre el concepto de estado del arte en la dimensión } \\
\text { del contexto de la organización. Igualmente, al hacer el comparativo, } \\
\text { el instrumento da respuesta al Decreto } 1072 \text { de 2015. Sin embargo, los } \\
\text { evaluadores refirieron que el instrumento podría dar alcance a todos los ítems } \\
\text { de evaluación del decreto y no solo a los de la SST. Referente a este ítem, es } \\
\text { importante decir que el objeto del instrumento y del presente proyecto está } \\
\text { direccionado solo a los tema de la GSST. }\end{array}$ \\
\hline 2 & $\begin{array}{l}\text { ¿El instrumento contempla parámetros que permiten } \\
\text { la evaluación de la GSST para los diferentes nichos } \\
\text { industriales (pymes y gran empresa) en Colombia? }\end{array}$ & $\begin{array}{l}\text { Se obtuvo un } 92,85 \% \text { de cumplimiento. Solo en uno de los casos se hacen } \\
\text { comentarios referentes al lenguaje para que el instrumento pueda ser trabajado } \\
\text { en las pymes. El instrumento fue ajustado y en los ítems genéricos, como los } \\
\text { de normatividad, se realiza claridad en los criterios sobre su cumplimiento, de } \\
\text { acuerdo con el caso para pequeña, mediana o gran empresa. }\end{array}$ \\
\hline 3 & $\begin{array}{l}\text { ¿El instrumento en su estructura semántica induce } \\
\text { y direcciona a la toma de decisiones en términos de } \\
\text { gestión? }\end{array}$ & $\begin{array}{l}\text { Se obtuvo un } 92,85 \% \text { de cumplimiento. Se realizaron ajustes a la semántica } \\
\text { del instrumento respecto a los términos de gestión, dado que en los supuestos } \\
\text { iniciales el lenguaje era netamente técnico y difícil para la comprensión en las } \\
\text { organizaciones, en especial en las pymes. }\end{array}$ \\
\hline 4 & $\begin{array}{l}\text { ¿El instrumento contempla variables para evaluar } \\
\text { la participación de niveles de alta gerencia, mandos } \\
\text { medios y trabajadores en el Sistema de Seguridad y } \\
\text { Salud en el Trabajo? }\end{array}$ & $\begin{array}{l}\text { Se obtuvo un } 100 \% \text { de cumplimiento. No hubo ajustes sobre este aspecto en el } \\
\text { instrumento. }\end{array}$ \\
\hline 5 & $\begin{array}{l}\text { ¿El uso del lenguaje es el adecuado y de fácil } \\
\text { comprensión? }\end{array}$ & $\begin{array}{l}\text { Se obtuvo un 92,85\% de cumplimiento. La redacción general del borrador } \\
\text { inicial del instrumento fue establecida de manera técnica, que a la luz del lector } \\
\text { en las organizaciones se consideró de alto nivel. Por ello, se revisó y ajustó el } \\
\text { instrumento a una redacción cotidiana sobre la SST. }\end{array}$ \\
\hline 6 & $\begin{array}{l}\text { ¿Todos los términos utilizados en el documento son } \\
\text { aplicables a la realidad colombiana? }\end{array}$ & Se obtuvo un $100 \%$ de cumplimiento. \\
\hline 7 & ¿La presentación del documento es adecuada? & $\begin{array}{l}\text { Se obtuvo un } 85,71 \% \text { de cumplimiento. El instrumento fue revisado en su } \\
\text { totalidad y se ajustó en la presentación adicionando la escala de medición. }\end{array}$ \\
\hline 8 & $\begin{array}{l}\text { ¿Las seis fases que contempla el instrumento son } \\
\text { claras, consecuentes y suficientes para medir la } \\
\text { gestión en materia de SST? }\end{array}$ & Se obtuvo un $100 \%$ de cumplimiento. \\
\hline 9 & $\begin{array}{l}\text { ¿Considera importante la inclusión tácita de variables } \\
\text { organizacionales para la medición de la GSST? }\end{array}$ & Se obtuvo un $100 \%$ de cumplimiento. \\
\hline 10 & $\begin{array}{l}\text { ¿El instrumento da respuesta al ciclo PHVA que es } \\
\text { la base fundamental del Draft de la ISO } 45001 \text { y del } \\
\text { Decreto } 1072 \text { de } 2015 \text { ? }\end{array}$ & Se obtuvo un $100 \%$ de cumplimiento. \\
\hline
\end{tabular}

Fuente: elaboración propia. 
Diseño y validación de contenido de un instrumento para medir la gestión de la seguridad y salud en el trabajo para organizaciones colombianas

Tabla 5. Calificaciones detalladas de la evaluación efectuada por parte de los expertos al intrumento

\begin{tabular}{|c|c|c|c|c|c|c|c|c|c|c|}
\hline TABLA DE DATOS & $\mathrm{P} 1$ & $\mathrm{P} 2$ & P3 & $\mathrm{P} 4$ & P5 & P6 & P7 & P8 & P9 & $\mathrm{P} 10$ \\
\hline Evaluador 01 & 1 & 1 & 1 & 1 & 1 & 1 & 1 & 1 & 1 & 1 \\
\hline Evaluador 02 & 1 & 1 & 1 & 1 & 1 & 1 & 1 & 1 & 1 & 1 \\
\hline Evaluador 03 & 1 & 1 & 1 & 1 & 1 & 1 & 1 & 1 & 1 & 1 \\
\hline Evaluador 04 & 1 & 1 & 1 & 1 & 1 & 1 & 1 & 1 & 1 & 1 \\
\hline Evaluador 05 & 1 & 1 & 1 & 1 & 1 & 1 & 1 & 1 & 1 & 1 \\
\hline Evaluador 06 & 1 & 1 & 1 & 1 & 1 & 1 & 1 & 1 & 1 & 1 \\
\hline Evaluador 07 & 0 & 1 & 1 & 1 & 1 & 1 & 0 & 1 & 1 & 1 \\
\hline Evaluador 08 & 0 & 1 & 1 & 1 & 1 & 1 & 0 & 1 & 1 & 1 \\
\hline Evaluador 09 & 0 & 1 & 0 & 1 & 0 & 1 & 0 & 1 & 1 & 1 \\
\hline Evaluador 10 & 1 & 1 & 1 & 1 & 1 & 1 & 1 & 1 & 1 & 1 \\
\hline Evaluador 11 & 0 & 1 & 1 & 1 & 1 & 1 & 1 & 1 & 1 & 1 \\
\hline Evaluador 12 & 0 & 1 & 1 & 1 & 0 & 1 & 1 & 1 & 1 & 1 \\
\hline Evaluador 13 & 1 & 1 & 1 & 1 & 1 & 1 & 1 & 1 & 1 & 1 \\
\hline Evaluador 14 & 1 & 1 & 1 & 1 & 1 & 1 & 1 & 1 & 1 & 1 \\
\hline Total & 9 & 14 & 13 & 14 & 12 & 14 & 11 & 14 & 14 & 14 \\
\hline
\end{tabular}

Fuente: elaboración propia.

\section{CONCLUSIONES}

Esta investigación permitió generar un instrumento para medir la GSST de acuerdo con el draft de la norma técnica ISO 45001, con validez de contenido dada por un grupo de expertos de las ARL de Colombia. Cabe precisar que esta propuesta de instrumentos es aplicable para la gran empresa y las Pyme.

Este instrumento, por estar ajustado a la realidad de los requerimientos evaluativos del Decreto 1072 de
2015, da respuesta a las necesidades de la gestión y la seguridad y salud en el trabajo, donde se resaltan las variables de cultura organizacional y participación de los trabajadores.

Las recomendaciones y los conceptos técnicos emitidos por el grupo de expertos fueron considerados en su totalidad y permitieron verificar la pertinencia, suficiencia y claridad de la herramienta de evaluación propuesta. Es importante destacar que esta herramienta 
da respuesta a las variables del draft de la norma técnica ISO 45001 y al Decreto 1072 de 2015, haciendo la salvedad de que solo se tuvieron en cuenta los ítems de evaluación correspondientes al Sistema de Gestión de la Seguridad en el Trabajo y no a la totalidad de la norma.

El instrumento propuesto en el ámbito empresarial aporta al diagnóstico de las variables intervinientes en el sistema de gestión de la seguridad y salud en el trabajo, y en la toma de decisiones que los empleadores puedan identificar, no solo con el objeto de contribuir al cumplimiento legal de la Ley 1562 del 2012 que opera para Colombia, sino también al bienestar y calidad de vida de la población trabajadora a través de planes de acción eficaces que tributen a los objetivos de la gestión.

Por otra parte, su aporte al campo investigativo se convertirá en un instrumento base para el desarrollo de nuevas investigaciones encaminadas al diseño de intervenciones en materia del sistema de gestión de seguridad y salud en el trabajo, y al seguimiento y control de estas de acuerdo con el mejoramiento continuo; base imprescindible para que las organizaciones inmersas en los sectores productivos sean rentables y sostenibles en un mundo globalizado.

Finalmente, las limitaciones de esta investigación y de la herramienta de evaluación del (SGSST) son la falta de validación de constructo y la no evaluación de su confiabilidad a través de pruebas estadísticas.

Es recomendable que en próximas investigaciones se considere su aplicación en organizaciones colombianas de distintos sectores de la economía, para poder evaluar el comportamiento del instrumento en cada uno de estos sectores. De igual modo, se requiere analizar el comportamiento de los ítems en organizaciones de distintos tamaños (considerando la clasificación por número de trabajadores establecida para Colombia).
De esta manera se podrá fijar la validación de constructo del instrumento diseñado para medir la gestión de la seguridad y salud en el trabajo para Colombia.

Dado que en la actualidad el Ministerio del Trabajo está adelantando un proyecto de estándares mínimos del SGSST para los empleadores, éstos deben tenerse en cuenta antes de someter a otros tipos de validación la herramienta de evaluación propuesta en esta investigación, con el ánimo de garantizar su coherencia con esos nuevos estándares.

Se sugiere que para desarrollar la aplicación en las organizaciones colombianas, el personal que la realice 0 que administre el proceso pertenezca al equipo de trabajo de SST, con el fin de que se garantice el entrenamiento mínimo de 50 horas de instrucción o niveles de formación formales educativos en la materia, para que así su experticia sea un aspecto positivo en la escala de valor del instrumento en el momento de verdad ante las organizaciones.

Este instrumento comparte el principio del draft de la norma técnica ISO 45001, por cuanto la aplicación de estos estándares son una herramienta de prevención en el control de riesgos; por tanto, se recomienda la participación activa del Comité Paritario de Seguridad y Salud en el Trabajo (COPASST), como ente administrador del proceso respecto a su aplicación e implementación de acciones de mejora.

Como resultado de la aplicación del instrumento, se sugiere que no se aborde la totalidad de las acciones de mejora para su implementación, sino que se identifiquen las variables prioritarias y poblaciones objeto de intervención, con el fin de que se controle y minimice la exposición al riesgo de la población expuesta.

Una vez se determinen los riesgos prioritarios de intervención y poblaciones objeto resultantes de la aplicación del instrumento, se recomienda hacer hincapié en 
el seguimiento y control de los sistemas de vigilancia epidemiológica para evaluar el mejoramiento continuo respecto a las condiciones de salud laboral de las organizaciones.

Se sugiere que el Plan de Acción Primaria, referente a la asesoría y apoyo ofertado por las ARL, se realice de manera consensuada y conjunta entre la empresa y la ARL, para que las variables de intervención den respuesta a los resultados de la aplicación del instrumento y no a actividades aisladas.

\section{REFERENCIAS}

British Standard Institution [BSI] (2007). OHSAS 18001:2007 Sistemas de gestión de seguridad y salud ocupacional. Londres: autor.

Cañadas, I. y Sánchez, A. (1998). Categorías de respuesta en escalas tipo Likert. Psicothema, 10(3), 623-631.

Colombia, Ministerio de Trabajo (2015). "Decreto 1072 de 2015". Bogotá, Colombia.

Colombia, Presidencia de la República. (2012). "Ley 1562 de 2012, 11 de julio de 2012”. Bogotá, Colombia.

Colombia, Presidencia de la República. (2014). "Decreto 1443 de 2014, 31 de julio de 2014”. Bogotá, Colombia.

Drais, E., Favaro, M. y Aubertain, G. (2008). Les systèmes de managements de la santé-sécurité en enterprise: Caractéristiques er conditions de mise en oewore. Paris: Institut National de Recherche et de Sécurité pour la prévention des accidents du travail et des maladies professionnelles.
Favaro, M. (2006). Dispositivo pedagógico para la concepción de indicadores de seguridad. En P. R. Mondelo, M. Mattila W. Karwowski y A. Hale (Eds.), Proceedings of the Fourth International Conference on Occupational Risk Prevention. Recuperado de https://www.prevencionintegral.com/canalorp/papers/orp-2006/dispositivo-pedagogico-paraconcepcion-indicadores-seguridad

Favaro, M. y Drais, E. (2007). Implementación de los sistemas de gestión de salud y seguridad en el trabajo. En Fifth International Conference on Occupational Risk Prevention. Santiago de Chile, Chile.

Fernández, B., Montes, J. y Vázquez, C. (2006). Desarrollo y validación de una escala de medición para el sistema de gestión de la seguridad laboral. Investigaciones Europeas de Dirección y Economía de la Empresa, 12(3), 77-93.

Fernández, R. (2007). Los distintos sistemas de gestión de la salud y seguridad en el trabajo. Gestión Práctica de Riesgos Laborales, (44).

Glaesel, K. (2014). Seguridad y salud ocupacional - la nueva ISO 45001. Recuperado de https:/l es.scribd.com/document/258988319/Seguridad -y-Salud-Ocupacional-Kristian-Glaesel

ISO. (2014). Anexo SL de las directivas de ISO/IEC, parte 1. ISO/IEC Directives, Part 1. Consolidated ISO Supplement - Procedures specific to ISO (Fifth edition). Recuperado de http://www.iso.org/sites/ directives/directives.html.

ISO. (2015). ISO/CD 45001.2. Occupational health and safety management systems - Requirements with guidance for use. ISO. Suiza: ISO.

Organización Internacional del Trabajo [OIT] (2001). Directrices relativas a los sistemas de gestión de la 
seguridad y salud en el trabajo. ILO-OHS. Ginebra: autor, Oficina Internacional de Ginebra.

Rodríguez, Y. (2012). Estrategias para el mejoramiento de la gestión de la salud y seguridad en el trabajo frente a las formas de vinculación en plantas de un grupo empresarial del sector industrial de Bogotá D.C. (Tesis de grado). Bogotá, Universidad Nacional de Colombia.

Rodriguez, Y. (2015). Documento Proyecto Diseño y Validación de un instrumento para medir la gestión de la Seguridad y Salud en el Trabajo para organizaciones colombianas. Documento de trabajo.
Maestría en Calidad y Gestión Integral. Bogotá: Universidad Santo Tomás.

Rodríguez, Y. y Molano, J. (septiembre-diciembre de 2012). Adaptación de una herramienta para la evaluación de la gestión de la salud y seguridad en el trabajo. El Hombre y la Máquina, (40), 7-21.

Rubio, J. (2001). Sistemas de gestión de la seguridad y salud en el trabajo. Sección Jurídica, 14(4), 4-13.

Salinas, R. (2008). Seguridad laboral y responsabilidad social corporativa. Recuperado el 16 de noviembre de 2015, de http://www.mapfre.com/fundacion/ html/revistas/seguridad/n109/articulo1.html 